Research Article

\title{
Graph of Fuzzy Topographic Topological Mapping in relation to $k$ - Fibonacci Sequence
}

\author{
Noorsufia Abd Shukor $\mathbb{D}^{1}$, Tahir Ahmad $\mathbb{D},{ }^{1}$ Amidora Idris, ${ }^{1}$ Siti Rahmah Awang $\left(\mathbb{D},{ }^{2}\right.$ \\ and Amirul Aizad Ahmad Fuad $\mathbb{D}^{3}$ \\ ${ }^{1}$ Department of Mathematical Sciences, Faculty of Science, Universiti Teknologi Malaysia, Johor Bahru 81310, Malaysia \\ ${ }^{2}$ Azman Hashim International Business School, Universiti Teknologi Malaysia, Johor Bahru 81310, Malaysia \\ ${ }^{3}$ Department of Mathematics, Xiamen University Malaysia, Jalan Sunsuria, Bandar Sunsuria 43900, Selangor, Malaysia
}

Correspondence should be addressed to Tahir Ahmad; tahir@utm.my

Received 18 July 2021; Accepted 9 August 2021; Published 24 August 2021

Academic Editor: Jia-Bao Liu

Copyright (c) 2021 Noorsufia Abd Shukor et al. This is an open access article distributed under the Creative Commons Attribution License, which permits unrestricted use, distribution, and reproduction in any medium, provided the original work is properly cited.

\begin{abstract}
A generated $n$-sequence of fuzzy topographic topological mapping, $\mathrm{FTTM}_{n}$, is a combination of $n$ number of FTTM's graphs. An assembly graph is a graph whereby its vertices have valency of one or four. A Hamiltonian path is a path that visits every vertex of the graph exactly once. In this paper, we prove that assembly graphs exist in $\mathrm{FTTM}_{n}$ and establish their relations to the Hamiltonian polygonal paths. Finally, the relation between the Hamiltonian polygonal paths induced from $\mathrm{FTTM}_{n}$ to the $k$ Fibonacci sequence is established and their upper and lower bounds' number of paths is determined.
\end{abstract}

\section{Introduction}

The fuzzy topographic topological mapping (FTTM) model is built to solve the neuromagnetic inverse problem proposed in 1999 [1]. It consists of four topological spaces, namely, magnetic contour plane (MC), base magnetic plane (BM), fuzzy magnetic field (FM), and topographic magnetic field (TM). The FTTM is developed to determine the location of a simulated neuromagnetic current source [2] as shown in Figure 1.

Later, Ahmad et al. [3] proved that the components of FTTM, namely, MC, BM, FM, and TM, were homeomorphic. The FTTM's structures and proofs of their homeomorphisms were outlined in [4].

Furthermore, FTTM can also be viewed as a sequence. The idea is possible when FTTM version 2 was successfully constructed by Rahman et al. [5] as shown in Figure 2. It was specially designed to solve the multiple current sources.
In 2006, Yun and Ahmad [4] noticed that if there are two elements of FTTM (see Figure 2), they will generate

$$
\left[\left(\begin{array}{l}
2 \\
1
\end{array}\right) \times\left(\begin{array}{l}
2 \\
1
\end{array}\right) \times\left(\begin{array}{l}
2 \\
1
\end{array}\right) \times\left(\begin{array}{l}
2 \\
1
\end{array}\right)\right]-2=14 \text { new elements of FTTM. }
$$

These 14 elements of FTTM are (MC, BM, FM, TMI), (MC, BM, FMI, TM), (MC, BMI, FM, TM), (MI, BM, FM, TM), (MC, BM, FMI, TMI), (MC, BMI, FMI, TM), (MI, BMI, FM, TM), (MI, BM, FM, TMI), (MI, BM, FMI, TM), (MC, BMI, FM, TMI), (MC, BMI, FMI, TMI), (MI, BM, FMI, TMI), (MI, BMI, FM, TMI), and (MI, BMI, FMI, TM). Further, Yun [6] conjectured the following.

Conjecture 1. If there exist $n$ elements of FTTM that are homeomorphic to each other componentwise, the number of new elements of FTTM that can be generated from these $n$ elements is 


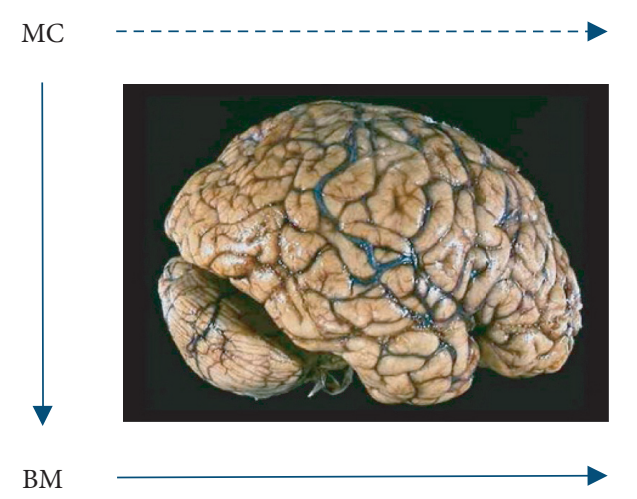

TM

Figure 1: FTTM model.

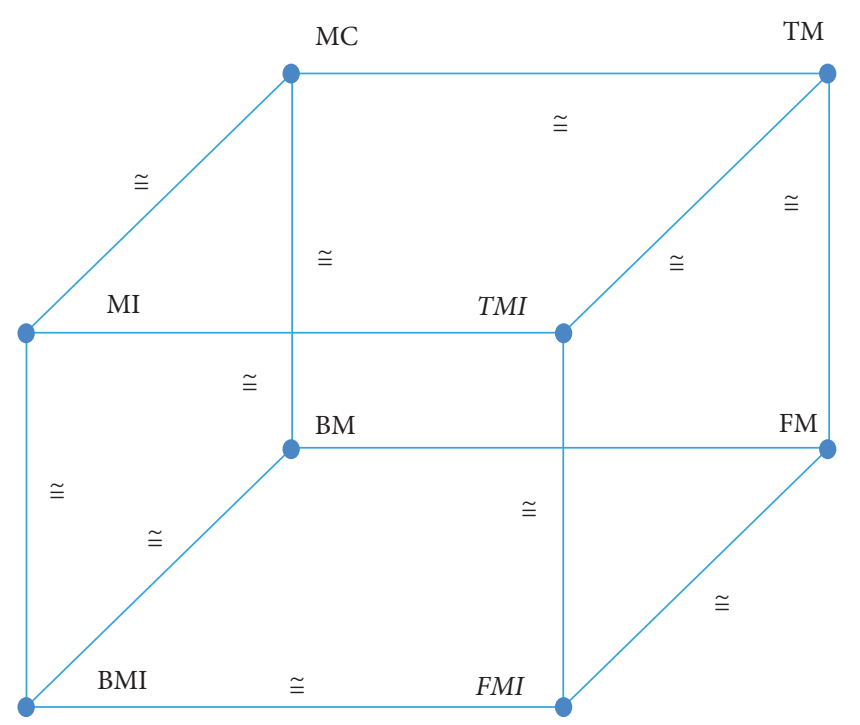

FIgURE 2: FTTM1 and FTTM2.

$$
\begin{aligned}
& {\left[\left(\begin{array}{l}
n \\
1
\end{array}\right) \times\left(\begin{array}{l}
n \\
1
\end{array}\right) \times\left(\begin{array}{c}
n \\
1
\end{array}\right) \times\left(\begin{array}{l}
n \\
1
\end{array}\right)\right]-n} \\
& =\left[\left(\frac{n !}{1 !(n-1) !}\right) \times\left(\frac{n !}{1 !(n-1) !}\right) \times\left(\frac{n !}{1 !(n-1) !}\right) \times\left(\frac{n !}{1 !(n-1) !}\right)\right]-n \\
& =[n \times n \times n \times n]-n \\
& =n^{4}-n .
\end{aligned}
$$

In order to prove the conjecture, Jamaian et al. [7] introduced the concept of sequence of FTTM as stated below.

Definition 1 (see [7]). Let $\mathrm{FTTM}_{i}=\left(\mathrm{MC}_{i}, \mathrm{BM}_{i}, \mathrm{FM}_{i}, \mathrm{TM}_{i}\right)$ such that $\mathrm{MC}_{i}, \mathrm{BM}_{i}, \mathrm{FM}_{i}, \mathrm{TM}_{i}$ are topological spaces with $\mathrm{MC}_{i} \cong \mathrm{BM}_{i} \cong \mathrm{FM}_{i} \cong \mathrm{TM}_{i}$. Set of $\mathrm{FTTM}_{i}$ is denoted by FTTM $=\left\{\right.$ FTTM $\left._{i}: i=1,2,3, \ldots, n\right\}$. Sequence of $n$ FTTM $_{i}$ of FTTM is FTTM $_{1}$, FTTM $_{2}$, FTTM $_{3}$, FTTM $_{4}, \ldots$, FTTM $_{n}$ such that $\mathrm{MC}_{i} \cong \mathrm{MC}_{i+1}, \mathrm{BM}_{i} \cong \mathrm{BM}_{i+1}, \mathrm{FM}_{i} \cong \mathrm{FM}_{i+1}$ and $\mathrm{TM}_{i} \cong \mathrm{TM}_{i+1}$.

A sequence of $n \mathrm{FTTM}_{i}$, without loss of generality, abbreviated as $\mathrm{FTTM}_{n}$, is illustrated in Figure 3.

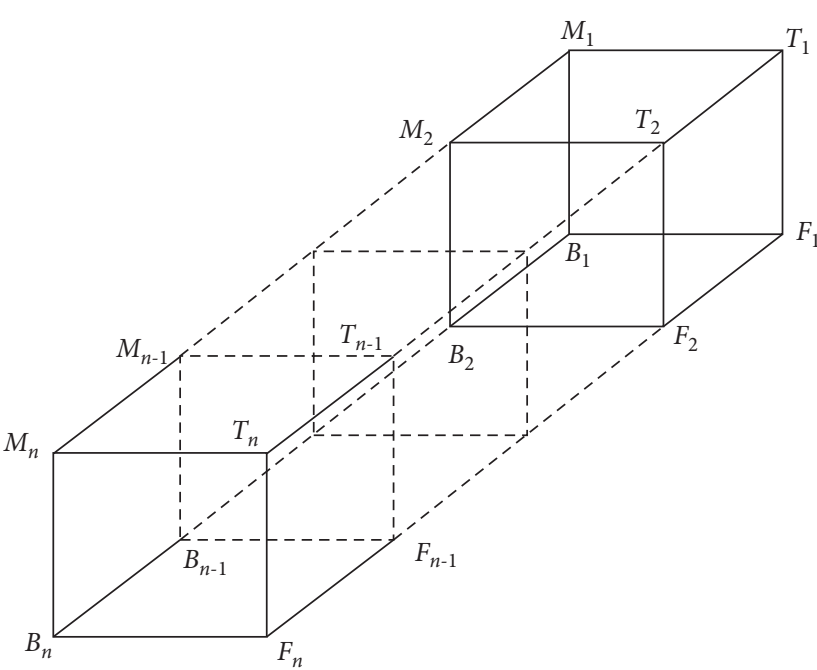

FIgURE 3: The sequence of $\mathrm{FTTM}_{n}$.

Finally, the conjecture was proven [7], and surprisingly the $\mathrm{FTTM}_{n}$ is related to the Pascal triangle.

Elsafi [8] then brought the concept of sequence of FTTM to another level. The researcher viewed and furnished sequence of FTTM as a graph. The details of the concept are presented in the following section.

\section{Graph of FTTM $_{\mathbf{n}}$}

Sayed and Ahmad introduced for the first time the representation of FTTM as a graph in [5]. Further, they defined the notion of order with respect to sequence of FTTM as follows.

Definition 2 (see [5]). Let $\mathrm{FTTM}^{n}=\left\{\mathrm{FTTM}_{1}, \mathrm{FTTM}_{2}\right.$, FTTM $_{3}, \ldots$, FTTM $_{n}$ \} be a sequence of $n$-FTTM (see Figure 3); then,

(1) $C_{i, j}$ FTTM $_{n}$ are cubes of order two that can be produced from the combination of FTTM $_{i}$ and FTTM $_{j}$ in FTTM $_{n}$ for $1 \leq i, j \leq n$ :

$$
\begin{aligned}
& i=\{1,2,3, \ldots, n-1\}, \\
& j=\{2,3, \ldots, n\} .
\end{aligned}
$$

(2) $\mid C_{i, j}$ FTTM $\left._{n}\right|_{1 \leq i<j \leq n}$ represent the number of cubes of order two that can be produced from the combination of $\mathrm{FTTM}_{i}$ and $\mathrm{FTTM}_{j}$ in $\mathrm{FTTM}_{n}$, such that $i \in I, j \in J, \forall i<j \leq n$.

Figure 4 shows the sequence of three terms of FTTM $_{3}$ such that FTTM $_{3}=\left\{\left(M_{1}, B_{1}, F_{1}, T_{1}\right),\left(M_{2}, B_{2}, F_{2}, T_{2}\right)\right.$, $\left.\left(M_{3}, B_{3}, F_{3}, T_{3}\right)\right\}$ and

$$
\begin{aligned}
C_{i, j} \text { FTTM }_{3}= & \left\{C_{1,2} \text { FTTM }_{3}, C_{2,3} \text { FTTM }_{3}, C_{1,3} \text { FTTM }_{3}\right\}, \\
& \mid C_{i, j} \text { FTTM }\left._{3}\right|_{1 \leq i<J \leq 3}=3 .
\end{aligned}
$$

Figure 5(a) shows that $\left(M_{1}, B_{1}, F_{3}, T_{3}\right)$ is an element of order two since its components appear in two terms of 


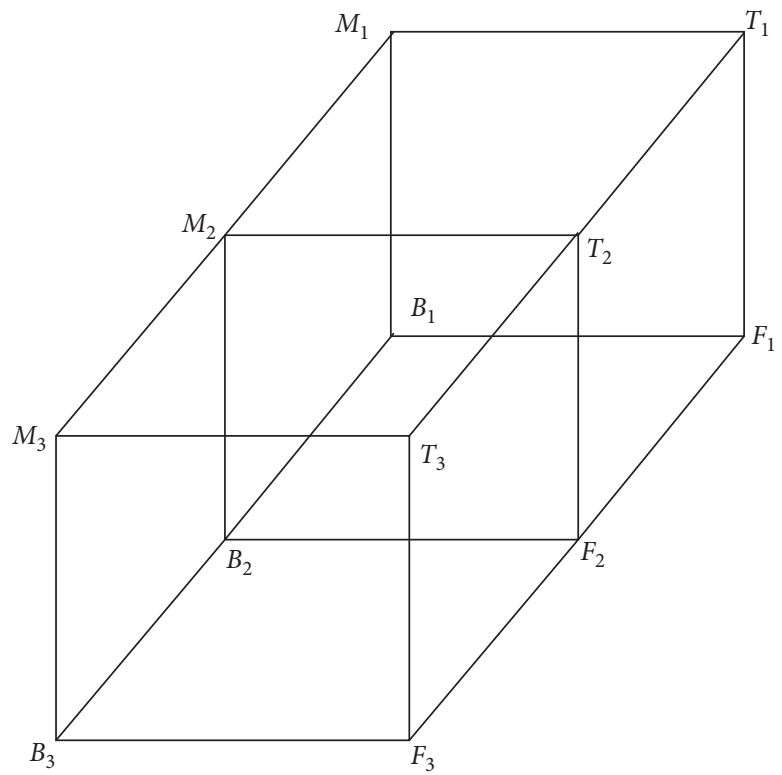

FIgURE 4: FTTM $_{3}$.

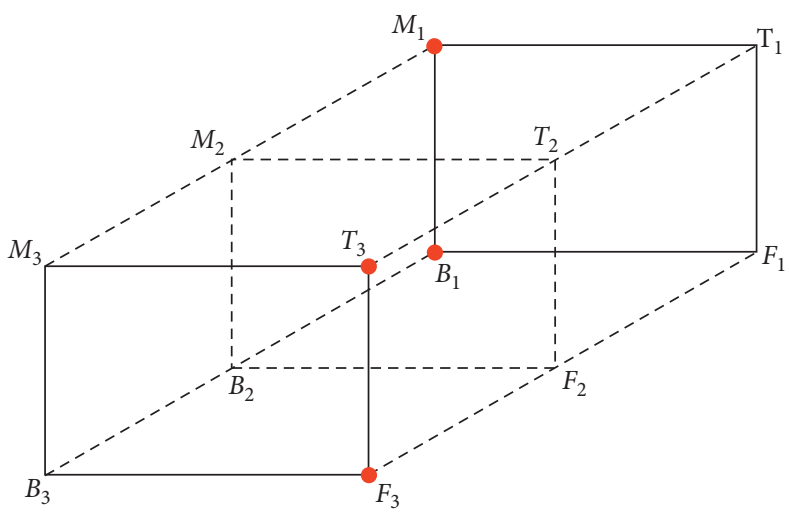

(a)

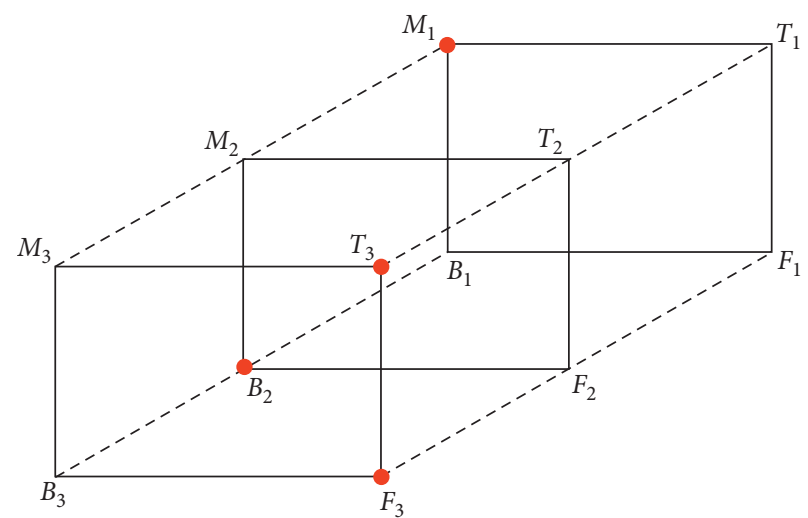

(b)

Figure 5: Example of $\mathrm{FTTM}_{3}$ with elements of different orders: (a) $\left(M_{1}, B_{1}, F_{3}, T_{3}\right)$; (b) $\left(M_{1}, B_{2}, F_{3}, T_{3}\right)$.

FTTM, namely, in FTTM ${ }_{1}$ and FTTM $_{3}$. By replacing $B_{1}$ with $B_{2}$, then $\left(M_{1}, B_{2}, F_{3}, T_{3}\right)$ is an element of order three since its components appear in $\mathrm{FTTM}_{1}, \mathrm{FTTM}_{2}$, and $\mathrm{FTTM}_{3}$ as presented in Figure 5(b).

Later, Ahmad et al. [9] established the relation of sequence of $\mathrm{FTTM}_{n}$ to $k$-Fibonacci sequence.

Theorem 1 (see [9]). The number of cubes produced by the combination of any three terms in FTTM ${ }_{n} ;$ FTTM $_{3 / n}$ can be presented as

$$
\begin{aligned}
\mathrm{FTTM}_{3 / n} & =\sum_{i=3}^{n}\left[\left(\begin{array}{c}
n+3-i \\
i
\end{array}\right)-\left(\begin{array}{c}
n+2-i \\
i+1
\end{array}\right)\right] \\
& =\frac{n(n-1)(n-2)}{3 !}, \quad \text { for } n \geq 3 .
\end{aligned}
$$

For examples, FTTM $_{3 / 1}=0$ for FTTM $_{1}$ (see Figure 1),

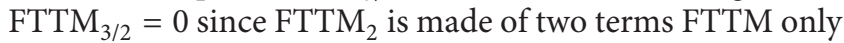
and FTTM $_{3 / 3}=1$ (see Figure 3). The numbers of FTTM for $n=1,2,3, \ldots, 10$ are summarized in Table 1 .

\section{Assembly Graph and Hamiltonian Path}

The concept of an assembly graph was first introduced by Angeleska et al. [10] for DNA structure through recombination process. The formal definition of an assembly graph is as follows.

Definition 3 (see [10]). An assembly graph is a finite connected graph, where all vertices are rigid vertices of valency 1 or 4. A vertex of valency 1 is called an end point. Let $\Gamma=$ $(V, E)$ be a finite graph with a set of vertices $V$ and a set of edges $E$. The number of 4 -valent vertices in $\Gamma$ is denoted with 
TABLE 1: FTTM $_{3 / n}$ for $n=1,2,3, \ldots, 10$

\begin{tabular}{lc}
\hline$n$ & FTTM $_{3 / n}$ \\
\hline$n=1$ & 0 \\
$n=2$ & 0 \\
$n=3$ & 1 \\
$n=4$ & 4 \\
$n=5$ & 10 \\
$n=6$ & 20 \\
$n=7$ & 35 \\
$n=8$ & 56 \\
$n=9$ & 84 \\
$n=10$ & 120 \\
\hline
\end{tabular}

$|\Gamma|$. The assembly graph is called trivial if $|\Gamma|=0$ (see Figure 6).

Angeleska et al. [10] also defined isomorphism between two assembly graphs. Basically, their isomorphism is a special case of the ordinary graph isomorphism.

Definition 4 (see [10]). Two assembly graphs $\Gamma_{1}=\left(V_{1}, E_{1}\right)$ and $\Gamma_{2}=\left(V_{2}, E_{2}\right)$ are isomorphic if there is a graph isomorphism $\Phi$ that preserves the cyclic order of each rigid vertex. More specifically, for a graph isomorphism $\Phi=\left(\Phi_{v}, \Phi_{e}: \Gamma_{1} \longrightarrow \Gamma_{2}\right)$ with $\Phi_{v}=V_{1} \longrightarrow V_{2}$ and $\Phi_{e}=E_{1} \longrightarrow E_{2}$, for every rigid vertex $\left(v,\left(e_{1}, e_{2}, e_{3}, e_{4}\right)^{\mathrm{cyc}}\right)$ in $\Gamma_{1}$, we have

$$
\begin{aligned}
& \left(\Phi_{v}(v),\left(\Phi_{v}\left(e_{1}\right), \Phi_{v}\left(e_{2}\right), \Phi_{v}\left(e_{3}\right), \Phi_{v}\left(e_{4}\right)\right)^{\mathrm{cyc}}\right) \\
& =\left(\Phi_{v}(v), E^{\mathrm{cyc}}\left(\Phi_{v}(v)\right)\right) .
\end{aligned}
$$

Angeleska et al. [10] then defined a composition operator for two assembly graphs. In particular, the initial vertex of $\Gamma_{1} \circ \Gamma_{2}$ is the initial vertex of $\Gamma_{1}$ and the terminal vertex of $\Gamma_{1} \circ \Gamma_{2}$ is the terminal vertex of $\Gamma_{2}$.

Definition 5 (see [10]). A composition $\Gamma_{1} \circ \Gamma_{2}$ of two (directed simple) assembly graphs $\Gamma_{1}$ and $\Gamma_{2}$ is the directed simple assembly graph, obtained by identifying the terminal vertex of $\Gamma_{1}$ with the initial vertex of $\Gamma_{2}$.

Furthermore, the following definitions yield some immediate properties for graph $\mathrm{FTTM}_{n}$.

Definition 6 (see [10]). Let $\Gamma$ be an assembly graph. An open path in $\Gamma$ is a homeomorphic image of the open interval $(0,1)$ in $\Gamma$. An open path is also represented by a sequence:

$$
\left(\left(e_{1} \backslash v_{0}\right), v_{1}, e_{2}, v_{2}, e_{3}, \ldots, v_{m-1}, e_{m}, v_{m},\left(e_{m+1} \backslash v_{m+1}\right)\right) \text {, }
$$

where $v_{i}^{\prime} s$ are vertices in $\Gamma$ for $i \in\{1,2, \ldots, m\}$ such that $v_{i} \neq v_{j}$ when $i \neq j$ and $e_{i}^{\prime} s$ are edges in $\Gamma$ for $i \in\{1,2, \ldots, m\}$ with endpoints $v_{i-1}$ and $v_{i}$, respectively, such that the initial vertex of $e_{1}$ (and possibly part of $e_{1}$ ) and the terminal vertex of $e_{m+1}$ (and possibly part of $e_{m+1}$ ) are not included.

An open path is a cycle if $e_{1}=e_{m+1}$.

Definition 7 (see [10]). A set of pairwise disjoint open paths $\left\{\gamma_{1}, \ldots, \gamma_{k}\right\}$ in $\Gamma$ is called Hamiltonian if their union contains all 4 -valent vertices of $\Gamma$. An open path $\gamma$ is called Hamiltonian if the set $\{\gamma\}$ is Hamiltonian.
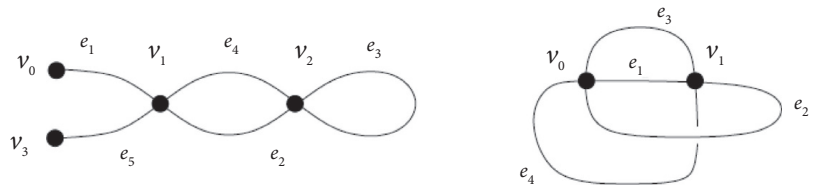

FIGURE 6: Examples of assembly graph [10].

Definition 8 (see [10]). Let $\Gamma$ be an assembly graph. The assembly number of $\Gamma$, denoted by $A n(\Gamma)$, is defined by $\operatorname{An}(\Gamma)=\min \{\mathrm{k} \mid$ there exists a Hamiltonian set of polygonal paths $\left\{\gamma_{1}, \ldots, \gamma_{k}\right\}$ in $\left.\Gamma\right\}$.

Definition 9 (see [10]). For a positive integer $m$, we define the minimal realization number for $m$ to be $R_{\min }(m) \min =$ $\{|\Gamma|: \operatorname{An}(\Gamma)=m\}$ where $|\Gamma|$ is the number of 4 -valent vertices in $\Gamma$. A graph $\Gamma$ such that $R_{\min }(m)=|\Gamma|$ is called a realization of $R_{\min }(m)$.

A Hamiltonian cycle is a cycle which passes through all vertices and the path ends at the initial vertex, and a Hamiltonian path is a path that visits every vertex of the graph exactly once.

A theorem that relates the number of Hamiltonian polygonal paths in an assembly graph is as follows.

Theorem 2 (see [11]). If $\Gamma$ is a simple assembly graph with $|\Gamma|=k$ and $C$ is the collection of all Hamiltonian polygonal paths of $\Gamma$, then

$$
|C| \leq F_{2 k+1}-1,
$$

where $F_{k}$ is the kth Fibonacci number.

\section{Assembly Graph of FTTM}

A graph of FTTM $_{n}$ as described above contains many subgraphs including assembly graphs. A new concept called maximal assembly graph for assembly subgraphs of $\mathrm{FTTM}_{n}$ is introduced.

Definition 10. Let $G_{1}, G_{2}, G_{3}, \ldots, G_{n}$ be subgraphs of $G$ $(V, E)$ whereby each $G_{i}$ is an assembly graph. A maximal assembly subgraph of $G_{i}$ is defined as $\left|\Gamma_{G_{i}}\right|=$ $\max \left\{\left|\Gamma_{G_{1}}\right|,\left|\Gamma_{G_{2}}\right|, \ldots,\left|\Gamma_{G_{n}}\right|\right\}$.

Table 2 lists all assembly subgraphs for $\mathrm{FTTM}_{3}$.

Let $\Gamma_{1}$ be the assembly subgraph as in Table $2(5)$ and $\Gamma_{2}$ be the assembly subgraph as in Table 2 (7); then $\left|\Gamma_{1} \circ \Gamma_{2}\right|=\left|\Gamma_{1}\right|+\left|\Gamma_{2}\right|=2+2=\operatorname{An}\left(\Gamma_{1}\right)+\operatorname{An}\left(\Gamma_{2}\right)$.

An $\mathrm{FTTM}_{4}$ produced 23 assembly subgraphs [12].

Then, consider $\Gamma_{1}=\left(V_{1}, E_{1}\right)$ and $\Gamma_{2}=\left(V_{2}, E_{2}\right)$ as assembly graphs as depicted in Figures $7(\mathrm{a})$ and $7(\mathrm{~b})$ whereby $V_{1}=\left\{B_{1}, B_{2}, M_{2}, F_{2}, B_{3}, M_{3}, F_{3}, B_{5}\right\}, \quad E_{1}=\left\{\left(B_{1}, B_{2}\right), \quad\left(B_{2}\right.\right.$, $\left.\left.M_{2}\right),\left(B_{2}, F_{2}\right),\left(B_{2}, B_{3}\right),\left(B_{3}, M_{3}\right),\left(B_{3}, F_{3}\right),\left(B_{3}, B_{4}\right)\right\} \quad$ and $V_{2}=\left\{T_{1}, T_{2}, M_{2}, F_{2}, T_{3}, M_{3}, F_{3}, T_{4}\right\}, \quad E_{2}=\left\{\left(T_{1}, T_{2}\right), \quad\left(T_{2}\right.\right.$, $\left.\left.F_{2}\right),\left(T_{2}, M_{2}\right),\left(T_{2}, T_{3}\right),\left(T_{3}, M_{3}\right),\left(T_{3}, F_{3}\right),\left(T_{3}, T_{4}\right)\right\}$, respectively.

Now, define $\Phi=\left(\Phi_{V}, \Phi_{E}: \Gamma_{1} \longrightarrow \Gamma_{2}\right)$ such that 
TABLe 2: Assembly subgraphs of $\mathrm{FTTM}_{3}$

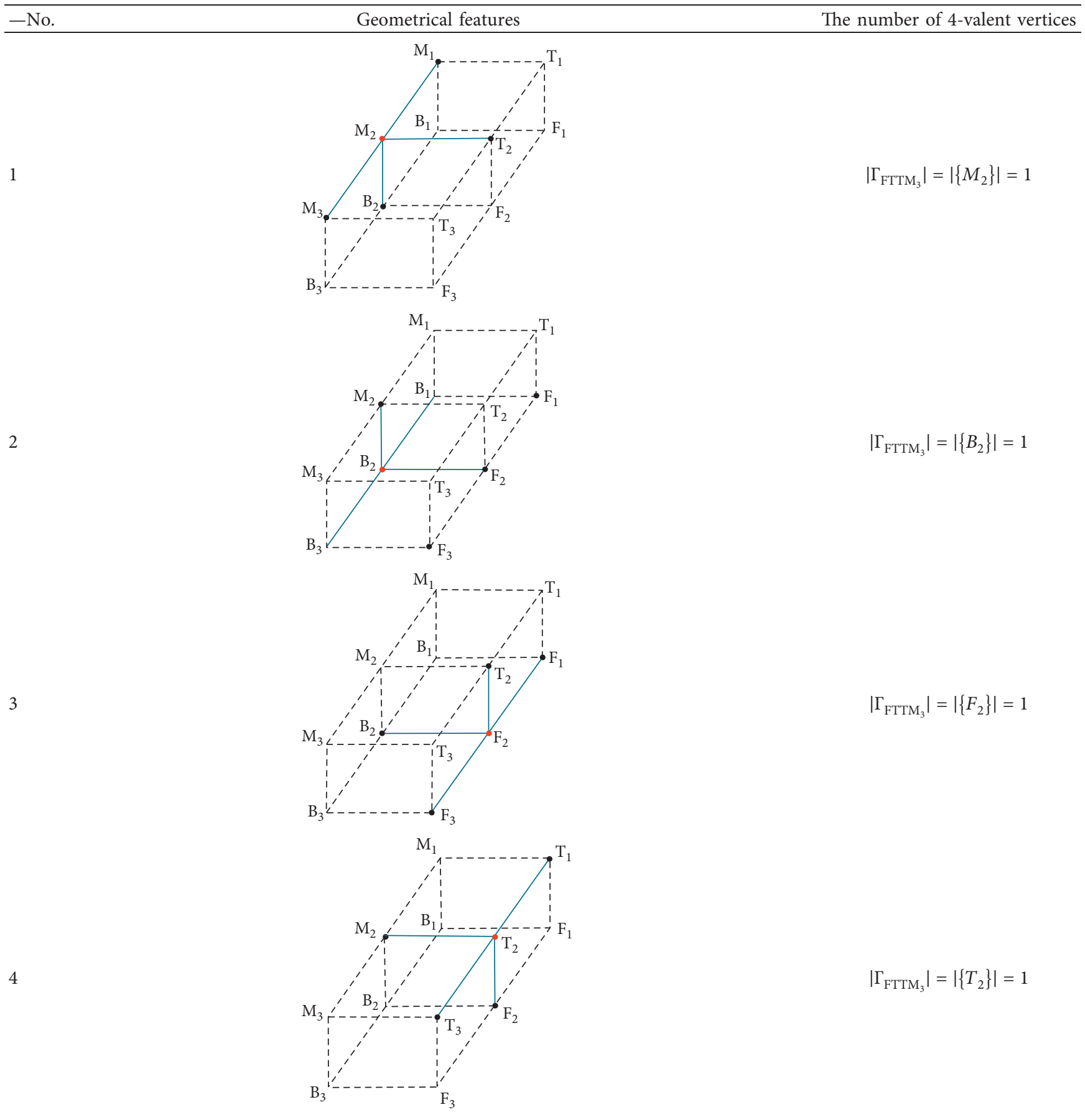


TABle 2: Continued.

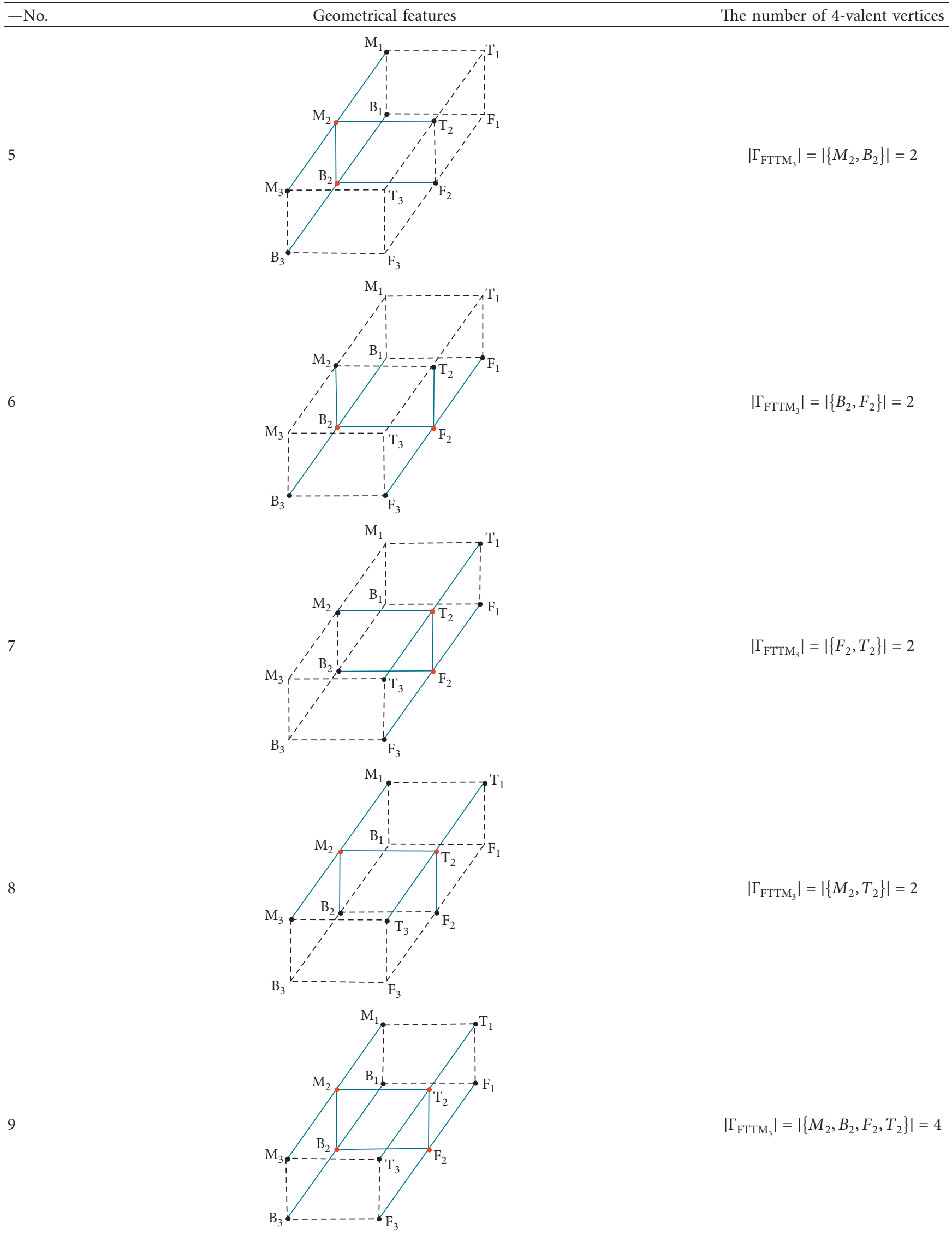




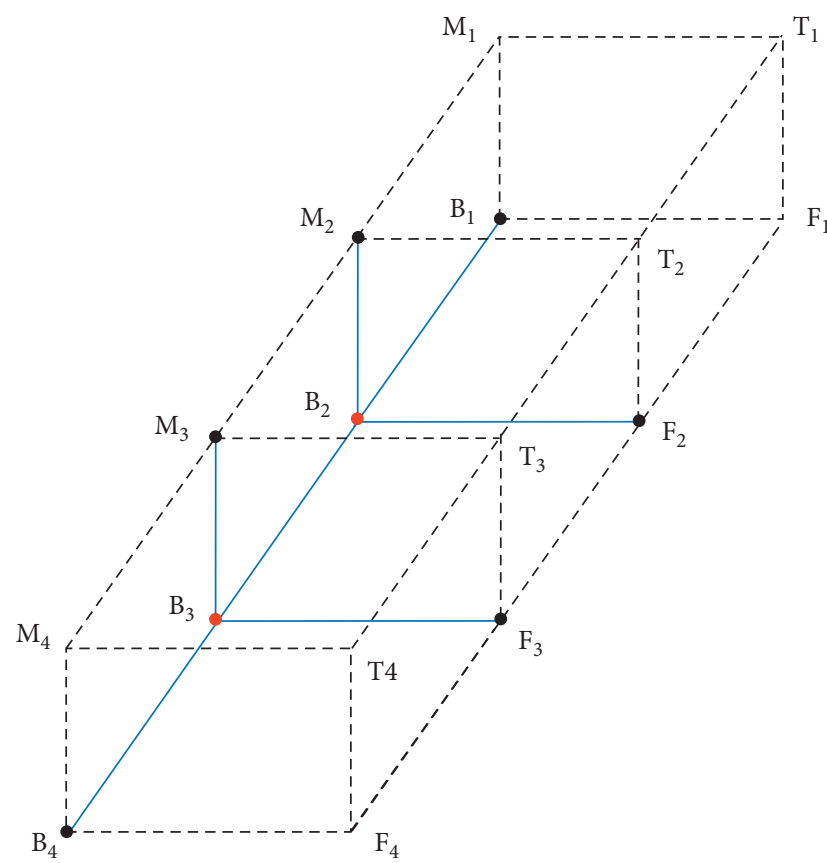

(a)

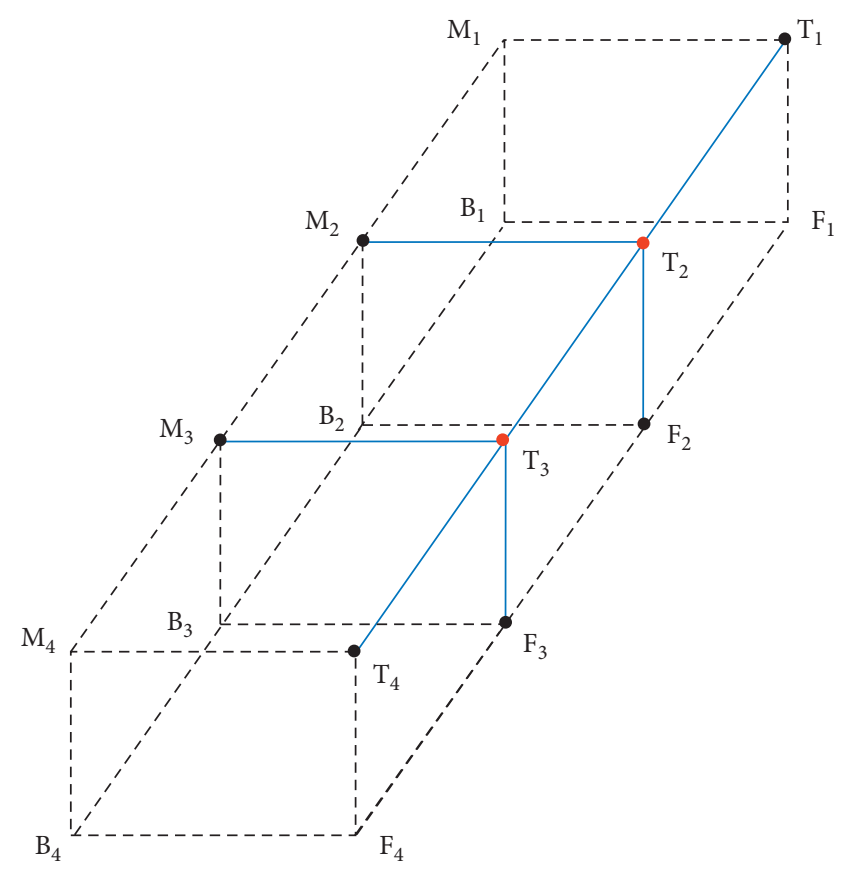

(b)

Figure 7: Example of $\mathrm{FTTM}_{4}$ with different 4-valent vertices.

$$
\begin{aligned}
\Phi_{v}\left(B_{1}\right) & =T_{1}, \\
\Phi_{E}\left(B_{1}, B_{2}\right) & =\left(T_{1}, T_{2}\right), \\
\Phi_{v}\left(B_{2}\right) & =T_{2}, \\
\Phi_{E}\left(B_{2}, M_{2}\right) & =\left(T_{2}, F_{2}\right), \\
\Phi_{v}\left(M_{2}\right) & =M_{2}, \\
\Phi_{E}\left(B_{2}, F_{2}\right) & =\left(T_{2}, M_{2}\right), \\
\Phi_{v}\left(F_{2}\right) & =F_{2}, \\
\Phi_{E}\left(B_{2}, B_{3}\right) & =\left(T_{2}, T_{3}\right), \\
\Phi_{v}\left(B_{3}\right) & =T_{3}, \\
\Phi_{E}\left(B_{3}, M_{3}\right) & =\left(T_{3}, M_{3}\right), \\
\Phi_{v}\left(M_{3}\right) & =M_{3}, \\
\Phi_{E}\left(B_{3}, F_{3}\right) & =\left(T_{3}, F_{3}\right), \\
\Phi_{v}\left(F_{3}\right) & =F_{3}, \\
\Phi_{E}\left(B_{3}, B_{4}\right) & =\left(T_{3}, T_{4}\right), \\
\Phi_{v}\left(B_{4}\right) & =T_{4} .
\end{aligned}
$$

Hence, $\Gamma_{1} \cong \Gamma_{2}$.

Clearly, a maximal assembly subgraph for $\mathrm{FTTM}_{n}$ is the resultant graph with edges for the first and the last terms of FTTM, in particular,FTTM 1 and FTTM $_{n}$ are neglected. The formal definition of a maximal assembly graph of FTTM $_{n}$ is stated.

Definition 11. The maximal assembly graph of $\mathrm{FTTM}_{n}$ is

$$
\Gamma_{\mathrm{FTTM}_{n}}=\mathrm{FTTM}_{n}-\left[E\left(\mathrm{FTTM}_{1}\right) \cup E\left(\mathrm{FTTM}_{n}\right)\right], \quad \text { for } n \geq 3,
$$

(a) $\left|\Gamma_{\text {FTTM }_{4}}\right|=\left|\left\{B_{2}, B_{3}\right\}\right|=2$. (b) $\left|\Gamma_{\text {FTTM }_{4}}\right|=\left|\left\{T_{2}, T_{3}\right\}\right|=2$.

and $\left|\Gamma_{\text {FTTM }_{n}}\right|$ is the number of its 4 -valent vertices.

From now on, the maximal assembly subgraph of

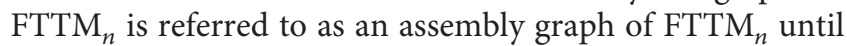
mentioned otherwise. Some properties on assembly graph of FTTM $_{n}$ for $n=3$ and 4 are summarized as follows.

Theorem 3 (see [13]). The FTTM 3 consists of an assembly subgraph.

Theorem 4 (see [13]). The FTTM 4 consists of an assembly subgraph.

The previous two results can be generalized to any FTTM $_{n}$.

Theorem 5 (see [13]). Every sequence of FTTM ${ }_{n}$ contains an assembly subgraph for $n \geq 3$.

Furthermore, Ahmad et al. [13] proved that the number of 4 -valent vertices of the maximal assembly graph, $\Gamma_{\text {FTTM }_{n}}$, is as follows.

Theorem 6 (see [13]). $\left|\Gamma_{\text {FTTM }_{n+2}}\right|=4+(n-1) 4$, for $n \in N$. The following theorems are immediate.

Theorem 7. Every sequence of FTTM $n$ yields minimal realization, $R_{\min }(m)$ number, for $n \geq 3$.

Proof. Theorem 5 guarantees that every sequence of FTTM ${ }_{n}$ contains an assembly subgraph for $n \geq 3$. An assembly graph for sequence of FTTM $_{n}$ is a maximal assembly graph by Definition 10 whereby $\left|\Gamma_{\text {FTTM }_{n}}\right|$ is the number of its 4 -valent 
vertices. By Definition 8 , every sequence of FTTM $_{n}$ yields minimal realization, $R_{\min }(m)$ number, for $n \geq 3$.

Theorem 8. $R_{\min }\left(\right.$ FTTM $\left._{n+2}\right)=4 n$ for sequence of FTTM ${ }_{n}$ and $n \in N$.

Proof. Theorem 7 guarantees that every sequence of FTTM ${ }_{n}$ yields minimal realization, $R_{\min }\left(\mathrm{FTTM}_{n}\right)$ number, for $n \geq 3$. Theorem 6 states that $\left|\Gamma_{\text {FTTM }_{n+2}}\right|=4+(n-1) 4$ for $n \in N$. Hence, $R_{\min }\left(\right.$ FTTM $\left._{n+2}\right)=4+(n-1) 4=4+4 n-4=4 n$ for sequence of $\operatorname{FTTM}_{n}$ and $n \in N$.

In fact, $\Gamma_{\mathrm{FTTM}_{n}}$ is a realization of $R_{\text {min }}\left(\mathrm{FTTM}_{n}\right)$ since $R_{\text {min }}\left(\right.$ FTTM $\left._{n}\right)=\left|\Gamma_{\mathrm{FTTM}_{n}}\right|$ for $n \geq 3$. Consequently, the following theorem is deduced.

Theorem 9. $R_{\min }\left(\operatorname{FTTM}_{n}\right)<R_{\min }\left(\right.$ FTTM $\left._{n+1}\right)$ for sequence of FTTM $_{n}$ and $n \geq 3$.

Proof.

$$
\begin{aligned}
R_{\min }\left(\operatorname{FTTM}_{n}\right) & =4 n, \text { By Theorem } 8 \\
& <4(n+1) \\
& <R_{\min }\left(\operatorname{FTTM}_{n+1}\right) \text { for sequence of } \text { FTTM }_{n} \text { and } n \geq 3 .
\end{aligned}
$$

\section{Hamiltonian Paths in an Assembly Graph of FTTM}

In previous section, we proved the existence of an assembly graph in any sequence of $\mathrm{FTTM}_{n}$ for $n \geq 3$. Hamiltonian polygonal paths exist in any assembly graph $\mathrm{FTTM}_{n}$ as well.

Theorem 10 (see [13]). $\Gamma_{\mathrm{FTTM}_{3}}$ consists of a set of Hamiltonian polygonal paths.

Theorem 11 (see [13]). $\Gamma_{\text {FTTM }_{4}}$ consists of a set of Hamiltonian polygonal paths.

In fact, the existence of Hamiltonian paths in any sequence of $\mathrm{FTTM}_{n}$ for $n \geq 3$ is generalized in the following theorem.

Theorem 12 (see [13]). $\Gamma_{F_{T T M}}$ consists of a set of Hamiltonian paths, for $n \geq 3$.

A coded program in [14] is modified to calculate the number of all Hamiltonian polygonal paths in an assembly graph of FTTM $_{n}$. Table 3 summarizes the number of Hamiltonian polygonal paths in assembly graphs of $\mathrm{FTTM}_{n}$ for $n=3,4,5, \ldots, 10$.

\section{Graph of FTTM $_{n}$ in Association to $k$ - Fibonacci Sequence}

The following theorem is the highlight of this paper. It links the work of Sayed and Ahmad [15] and Ahmad et al. [9], i.e., the relation of graph of FTTM and Fibonacci number (see Figure 8).
Theorem 13 (see [12]). Let FTTM ${ }_{n}$ be a sequence of n-FTTM with $\left|\Gamma_{\text {TTTM }_{n}}\right|=k$ and $C$ be the collection of all sets of Hamiltonian polygonal paths of FTTM ${ }_{n}$; then,

$$
|C| \leq F_{2 k+1}-1
$$

where $F_{k}$ is the kth Fibonacci number.

Proof. Let FTTM $n$ be a sequence of $n$-FTTM. By Theorem 5, FTTM $_{n}$ consists of assembly graphs, namely, $\Gamma_{\text {FTTM }_{n}}$. Then, Theorem 12 guarantees that $\Gamma_{\mathrm{FTTM}_{n}}$ consists of a set of Hamiltonian polygonal paths, say $C$. Using Theorem 2, for FTTM $_{n},|C| \leq F_{2 k+1}-1$ as required whereby $F_{k}$ is the $k$ th Fibonacci number.

Thus, the connections illustrated in Figure 8 are completed. A refinement of Theorem 13 is given in the following corollary.

Corollary 14 (see [12]). Let FTTM $_{n}$ be a sequence of $n$ FTTM for $n \geq 3$ and $C$ be the set of all Hamiltonian polygonal paths of FTTM, then,

$$
|C| \leq F_{8 n+1}-1 .
$$

Proof. Let $\mathrm{FTTM}_{n}$ be a sequence of $n$-FTTM for $n \geq 3$. By Theorem 5, FTTM $_{n}$ consists of assembly graphs, namely, $\Gamma_{\mathrm{FTTM}_{n}}$. Further, Theorem 6 reveals that $\left|\Gamma_{\text {FTTM }_{n+2}}\right|=4+(n-1) 4$, for $n \in N$. Theorem 12 guarantees that $\Gamma_{\mathrm{FTTM}_{n}}^{n+2}$ consists of a set $C$, that is, all its Hamiltonian polygonal paths. By replacing $k=4+(n-1) 4$, for $n \in N$, in Theorem 13,

$$
\begin{aligned}
|C| & \leq F_{2 k+1}-1 \\
& =F_{2(4+(n-1) 4)+1}-1, \text { replace } k=4+(n-1) 4 \\
& =F_{2(4+4 n-4)+1}-1 \\
& =F_{2(4 n)+1}-1 \\
& =F_{8 n+1}-1 .
\end{aligned}
$$

Table 4 lists Hamiltonian polygonal paths of $\mathrm{FTTM}_{n}$ in relation to $k$-Fibonacci numbers for $n=3$ to 10 .

The following theorem highlights the lower and upper bounds for Hamiltonian polygonal paths of $\mathrm{FTTM}_{n}$.

Theorem 15. Let FTTM $M_{n}$ be a sequence of $n$-FTTM for $n \geq 3$ and $C$ be the set of all Hamiltonian polygonal paths of FTTM $_{n}$; then,

$$
\frac{n(n-1)(n-2)}{3 !} \leq|C| \leq F_{8 n+1}-1 .
$$

Proof. Definition 11 states that $\left|\Gamma_{\mathrm{FTTM}_{n}}\right|$ is the number of 4valent vertices. These 4 -valent vertices can only exist only for (at least) three terms of FTTM, i.e., FTTM $_{3 / n}$. But Theorem 1 guarantees that the number of cubes produced by the combination of any three terms in $\mathrm{FTTM}_{n}$; $\mathrm{FTTM}_{3 / n}$ is $(n(n-1)(n-2) / 3 !)$. In other words, the lower bound of Hamiltonian polygonal paths of $\mathrm{FTTM}_{n}, C$, is obtained as $|C| \geq\left(n(n-1)(n-2) / 3\right.$ !). Corollary 14 states $|C| \leq F_{8 n+1}-1$ for $n \geq 3$. Hence, $(n(n-1)(n-2) / 3 !) \leq|C| \leq F_{8 n+1}-1$ as required. 
TABLE 3: Hamiltonian polygonal paths in assembly graphs of $\mathrm{FTTM}_{n}$ for $n=3,4,5, \ldots, 10$

\begin{tabular}{|c|c|c|c|}
\hline FTTM $_{n}$ & No. of vertices & No. of 4-valent vertices & Hamiltonian polygonal paths \\
\hline $\mathrm{FTTM}_{3}$ & 12 & 4 & 8 \\
\hline $\mathrm{FTTM}_{4}$ & 16 & 8 & 144 \\
\hline $\mathrm{FTTM}_{5}$ & 20 & 12 & 1,168 \\
\hline FTTM $_{6}$ & 24 & 16 & 8,032 \\
\hline FTTM $_{7}$ & 28 & 20 & 49,312 \\
\hline $\mathrm{FTTM}_{8}$ & 32 & 24 & 281,248 \\
\hline FTTM $_{9}$ & 36 & 28 & $1,523,920$ \\
\hline FTTM $_{10}$ & 40 & 32 & $7,953,408$ \\
\hline
\end{tabular}

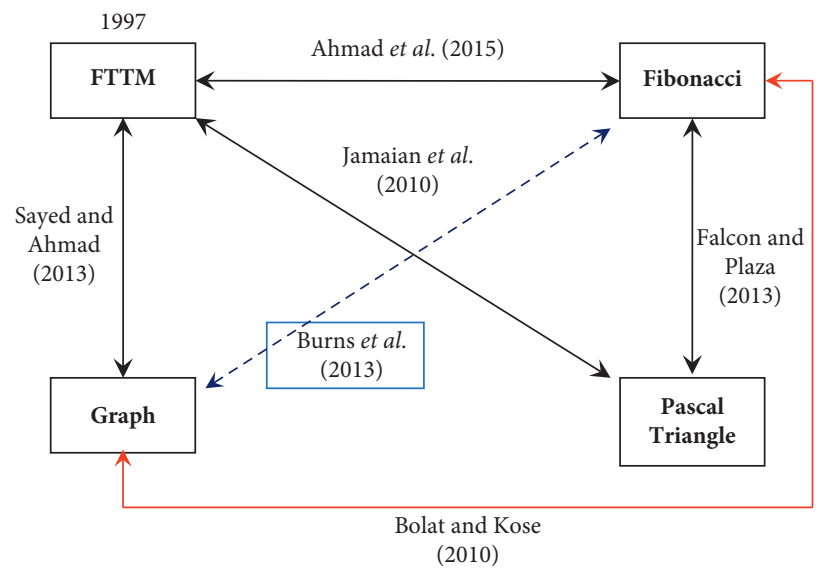

FIgURE 8: Three mathematical concepts with respect to FTTM.

TABLE 4: Hamiltonian polygonal paths and $F_{2 k+1}$ for $n=3$ to 10 .

\begin{tabular}{|c|c|c|c|}
\hline$\left|\Gamma_{\text {FTTM }_{n}}\right|=\mathbf{k}$ & $\mathbf{F}_{2 \mathbf{k}+1}$ & $|\mathrm{C}|$ & $|\mathbf{C}| \leq \mathbf{F}_{2 \mathbf{k}+1}-1$ \\
\hline$\left|\Gamma_{\text {FTTM }_{3}}\right|=4$ & $F_{2(4)+1}=F_{9}=34$ & 8 & $8 \leq 33$ \\
\hline$\left|\Gamma_{\mathrm{FTTM}_{4}}\right|=8$ & $F_{2(8)+1}=F_{17}=1,597$ & 144 & $144 \leq 1,596$ \\
\hline$\left|\Gamma_{\text {FTTM }_{5}}\right|=12$ & $F_{2(12)+1}=F_{25}=75,025$ & 1,168 & $1,168 \leq 75,024$ \\
\hline$\left|\Gamma_{\text {FTTM }_{6}}\right|=16$ & $F_{2(16)+1}=F_{33}=3,524,578$ & 8,032 & $8,032 \leq 3,524,577$ \\
\hline$\left|\Gamma_{\text {FTTM }_{7}}\right|=20$ & $F_{2(20)+1}=F_{41}=156,580,141$ & 49,312 & $49,312 \leq 165,580,140$ \\
\hline$\left|\Gamma_{\text {FTTM }_{8}}\right|=24$ & $F_{2(24)+1}=F_{49}=7,778,742,049$ & 281,248 & $281,248 \leq 7,778,742,048$ \\
\hline$\left|\Gamma_{\text {FTTM }_{9}}\right|=28$ & $F_{2(28)+1}=F_{57}=365,435,296,162$ & $1,523,920$ & $1,523,920 \leq 365,435,296,161$ \\
\hline$\left|\Gamma_{\mathrm{FTTM}_{10}}\right|=32$ & $F_{2(32)+1}=F_{65}=17,167,680,177,565$ & $7,953,408$ & $7,953,408 \leq 17,167,680,177,564$ \\
\hline
\end{tabular}

TABLE 5: Lower and upper bounds for Hamiltonian polygonal paths of $\mathrm{FTTM}_{n}$ for $n=3$ to 10 .

\begin{tabular}{lccc}
\hline FTTM $_{\mathbf{n}}$ & FTTM $_{3 / \mathbf{n}}$ & $\mid \mathbf{C}$ & $\mathbf{F}_{8 \mathbf{n}+1}-1$ \\
\hline FTTM $_{3}$ & 1 & 8 & 33 \\
FTTM $_{4}$ & 4 & 144 & 1,596 \\
FTTM $_{5}$ & 10 & 1,168 & 75,024 \\
FTTM $_{6}$ & 20 & 8,032 & $3,524,577$ \\
FTTM $_{7}$ & 35 & 49,312 & $165,580,140$ \\
FTTM $_{8}$ & 56 & 281,248 & $7,778,742,048$ \\
FTTM $_{9}$ & 84 & $1,523,920$ & $365,435,296,161$ \\
FTTM $_{10}$ & 120 & $7,953,408$ & $17,167,680,177,564$ \\
\hline
\end{tabular}

The following table (see Table 5) lists the lower and upper bounds for Hamiltonian polygonal paths in $\mathrm{FTTM}_{n}$ for $n=3$ to 10 .

\section{Conclusions}

The aim of this paper is to prove that there exists a relationship between $\mathrm{FTTM}_{n}$ (sequence of $n$-FTTM) and $k$ Fibonacci sequence. We have established the lower and upper bounds for the established relation.

\section{Data Availability}

No data were used to support this study.

\section{Conflicts of Interest}

The authors declare that they have no conflicts of interest. 


\section{Acknowledgments}

The authors acknowledge the support of Universiti Teknologi Malaysia (UTM) and Ministry of Higher Education Malaysia (MOHE). This research was also supported by Fundamental Research Grant Scheme (FRGS) (FRGS/1/ 2020/STG06/UTM/01/1), Ministry of Education, Malaysia.

\section{References}

[1] T. Ahmad, R. Shah Ahmad, F. Zakaria, and L. L. Yun, "Development of detection model for neuromagnetic fields," in Proceedings of the BIOMED 2000, pp. 119-121, Universiti Malaya, Kuala Lumpur, Malaysia, 2000.

[2] T. Ahmad, R. S. Ahmad, W. E. A. W. Abdul Rahman, L. L. Yun, and F. Zakaria, "Fuzzy topographic topological mapping for localisation simulated multiple current sources of MEG," Journal of Interdisciplinary Mathematics, vol. 11, no. 3, pp. 381-393, 2008.

[3] T. Ahmad, R. Shah Ahmad, L. L. Yun, F. Zakaria, and W. E. Z. Rahman, "Homeomorphisms of fuzzy topographic topological mapping (FTTM)," Matematika, vol. 21, no. 1, pp. 34-42, 2005.

[4] L. L. Yun and T. Ahmad, "Pembinaan homeomorfisma dari sfera ke elipsoid," Matematika, vol. 19, no. 2, pp. 121-138, 2003.

[5] W. E. Z. W. A Rahman, T. Ahmad, and R. Shah Ahmad, "Simulating the neuronal current sources in the brain," in Proceedings of the BIOMED 2002, pp. 19-22, University Malaya, Antalya, Turkey, September 2002.

[6] L. L. Yun, "Group-like algebraic structures of fuzzy topographic topological mapping for solving neuromagnetic inverse problem" Ph.D. thesis, University Teknologi Malaysia, Johor Bahru, Malaysia, 2006.

[7] S. S. Jamaian, T. Ahmad, and J. Talib, "Generalized finite sequence of fuzzy topographic topological mapping," Journal of Mathematics and Statistics, vol. 6, no. 2, pp. 151-156, 2010.

[8] M. S. A. E. Elsafi, "Combinatorial analysis of $\mathrm{N}$-tuple polygonal sequence of fuzzy topographic topological mapping" Ph.D. thesis, Universiti Teknologi Malaysia, Johor Bahru, Malaysia, 2014.

[9] T. Ahmad, A. A. M. Yunus, and N. A. A. Karim, "Sequence of fuzzy topographic topological mapping and $k$-fibonacci sequence," Applied Mathematical Sciences, vol. 9, no. 11, pp. 533-550, 2015.

[10] A. Angeleska, N. Jonoska, and M. Saito, "DNA recombination through assembly graphs," Discrete Applied Mathematics, vol. 157, no. 14, pp. 3020-3037, 2009.

[11] J. Burns, E. Dolzhenko, N. Jonoska, T. Muche, and M. Saito, "Four-regular graphs with rigid vertices associated to DNA recombination," Discrete Applied Mathematics, vol. 161, no. 10-11, pp. 1378-1394, 2013.

[12] N. A. Shukor, "Combinatorial structure of cube and $k$-fibonacci sequence," Master thesis, Universiti Teknologi Malaysia, Johor Bahru, Malaysia, 2020.

[13] T. Ahmad, N. A. Shukor, A. Idris, and Z. Mahmud, "Hamiltonian polygonal path in assembly graph of FTTM," AIP Conference Proceedings, vol. 2184Penang, Malaysia, Article ID 0200002, 2019.

[14] Z. Mahamud and T. Ahmad, "Generated paths of an autocatalytic set of a secondary system of a pressurized water reactor," Malaysian Journal of Fundamental and Applied Sciences, vol. 13, no. 2, pp. 91-94, 2017.
[15] M. Sayed and T. Ahmad, "Graph of finite sequence of fuzzy topographic topological mapping of order two," Journal of Mathematics and Statistics, vol. 9, no. 1, pp. 18-23, 2013. 